\title{
Structural Design Exploration of an Electric Powered Multi-Propulsor Wing Configuration
}

\author{
James B. Moore ${ }^{1}$ \\ And \\ Steve Cutright ${ }^{2}$ \\ NASA Langley Research Center, Hampton, VA, 23681
}

\begin{abstract}
Advancements in aircraft electric propulsion may enable an expanded operational envelope for electrically powered vehicles compared to their internal combustion engine counterparts. High aspect ratio wings provide additional lift and drag reduction for a proposed multi-propulsor design, however, the challenge is to reduce the weight of wing structures while maintaining adequate structural and aeroelastic margins. Design exploration using a conventional design-and-build philosophy coupled with a finite element method (FEM)-based design of experiments (DOE) strategy are presented to examine high aspect ratio wing structures that have spanwise distributed electric motors. Multiple leading-edge-mounted engine masses presented a challenge to design a wing within acceptable limits for dynamic and aeroelastic stability. Because the first four primary bending eigenmodes of the proposed wing structure are very sensitive to outboard motor placement, safety-of-flight requirements drove the need for multiple spars, rib attachments, and outboard structural reinforcements in the design. Global aeroelasticity became an increasingly important design constraint during the on-going design process, with outboard motor pod flutter ultimately becoming a primary design constraint. Designers successively generated models to examine stress, dynamics, and aeroelasticity concurrently. This research specifically addressed satisfying multidisciplinary design criteria to generate fluid-structure interaction solution sets, and produced high aspect ratio primary structure designs for the NASA Scalable Convergent Electric Propulsion Technology and Operations Research (SCEPTOR) project in the Aeronautic Research Mission Directorate at NASA. In this paper, a dynamics-driven, quasi-inverse design methodology is presented to address aerodynamic performance goals and structural challenges encountered for the SCEPTOR demonstrator vehicle. These results are compared with a traditional computer aided design based approach.
\end{abstract}

\section{Introduction}

The use of distributed leading-edge-mounted electric motors enables a conventional wing to operate at greatly elevated lift coefficients $\left(\mathrm{C}_{1}\right)$, providing designers with the ability to develop aircraft with reduced fuel operating costs compared with their internal combustion engine counterparts. The NASA Convergent Aeronautical Solutions (CAS) leading edge asynchronous propulsor Technology (CAS-LeapTech) project seeks to demonstrate that this approach (shown in Figure 1), can increase vehicle efficiency by nearly five times compared to a conventional aircraft. Primary structures, such as the wing, are critical structures that would result in a loss of vehicle if a failure occurs. Therefore, the wing primary structural design is the focus of this study and the objective was to develop a low mass design with adequate structural margins for a planned flight test vehicle denoted as Scalable Convergent Electric Propulsion Technology and Operations Research (SCEPTOR). Reduction in mass is critical to reduce liftinduced drag and results in a reduction of specific energy consumption through aerodynamic efficiency. ${ }^{1}$ Both static margins and dynamic stability were addressed for SCEPTOR by designing wing structures using a quasi-inverse

\footnotetext{
${ }^{1}$ Research Aerospace Engineer, LaRC, Structural Mechanics and Concepts Branch, Mail Stop 190

${ }^{2}$ Research Aerospace Engineer, LaRC, Structural and Thermal Systems Branch, Mail Stop 431
} 
design method, rather than the traditional approach of developing detailed computer aided design (CAD) design with limited aerodynamic inputs to the fluid structure-interaction (FSI) problem. Although the SCEPTOR structure is not an explicit inverse FSI design, which can derive structure from load sets, it is representative of a multidisciplinary analytical collaboration with a commercial design group, Xperimental LLC, to develop structural concepts. For the SCEPTOR program, this approach avoided the initial laborious process of an iterative CAD redesign cycle currently in commercial practice. The quasi-inverse process used also permits the parallel validation of model sets between partners to ensure common design space definition and to prevent disparities in dynamics behavior, that were later discovered, from negatively impacting structural margin targets. Developing the analytical model to be used in a design of experiments (DOE) study prior to CAD definition provides a reference point for future design space exploration and structural correlation studies that ultimately impact the final detailed design.

The dynamics spectrum of the SCEPTOR structure is complicated as a result of the motors being placed on the leading edge of the wing. The positioning of the motor masses on high aspect ratio (AR) designs ${ }^{2}$ significantly affects the displacement amplitude, frequency ratios and frequency coupling. Preliminary results indicate that the location of engine masses has a critical impact on structural stability margins. As mass increases in the outboard locations, structural stability margins tend to decrease dramatically due to lower natural frequencies which can be more susceptible to flutter. These masses also result in large deflections and the tendency for frequency coupling between modes. For this configuration, the outboard motor pods demonstrate nominal aeroelastic stability from whirl flutter due to the low section modulus of the wing compared with a conventional pylon arrangement. However, advanced composite materials and construction techniques enable the aggressive pylon design for this unique configuration, where the heavier outboard propulsor is designed to maintain cruise performance with tipvortex abatement. ${ }^{3}$

Composite materials were selected over conventional aluminum to enable designs with complex, compound curvature to minimize aerodynamic drag. This material choice also eliminated the need for expensive machine tooling and associated jig hardware to promote a rapid development cycle and minimize costs. Due to the potential for large deflections and blade liberation on inboard motor pods ${ }^{4}$, a dual-load path stressed skin arrangement utilizing a carbon fiber material system was chosen for pod and skin acreage. Unidirectional fiber was added to spar caps to increase section modulus, minimize deflection and increase fundamental frequency modes which can enhance flutter margins.

A span-wise load distribution (SLD) for a conventional wing is elliptical with a near-zero lift coefficient (Cl) at the tip which degrades planform efficiency and reduces bending load at lower aspect ratios. The SCEPTOR propulsor wing configuration is responsible for reducing the tip roll-up vortices losses using the outboard motor pod and produces an SLD with much higher $\mathrm{Cl}$ distribution at lower airspeeds by increasing local dynamic pressure with high-lift motors air flow. The structural challenge is to accommodate a large $(120 \mathrm{lbm})$ cantilevered electric cruise motor, with $400+\mathrm{lbf}$ static sea level thrust, at the wing tips and preserve aeroelastic stability margins for an optimized thin section airfoil per guidance from Federal Aviation Administration (FAA) advisory circular (AC) 23.629. ${ }^{5}$ 


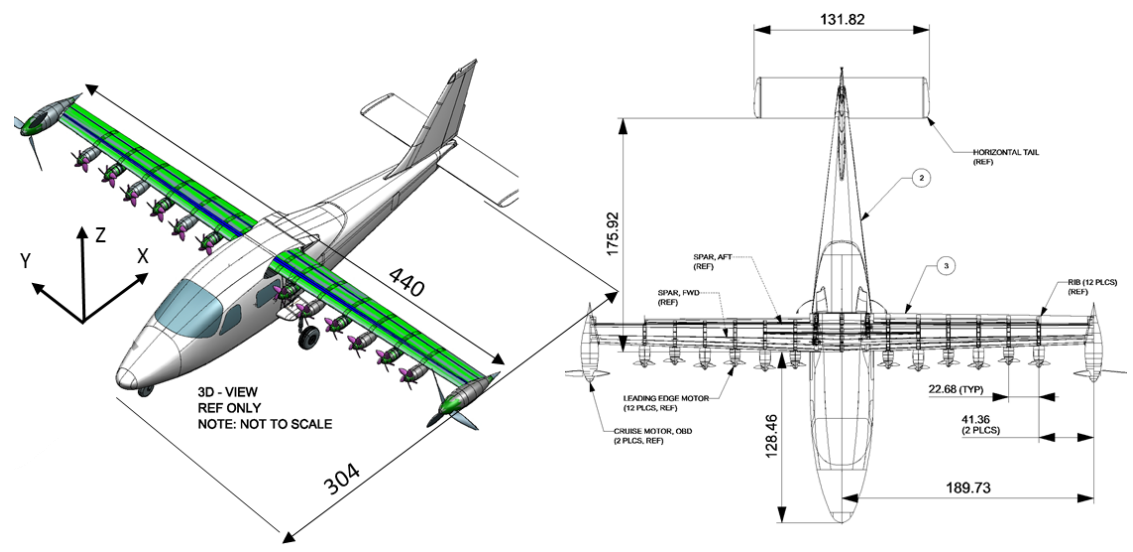

Figure 1: SCEPTOR vehicle isometric view (approximate dimensions are in inches)

Several structural configurations were investigated parametrically to include variations in spar, rib, and stiffener placement. Due to the susceptibility of flutter in this high aspect ratio configuration, three spars were ultimately incorporated to increase bending resistance, provide fuselage attachment locations and add local buckling resistance at the fuselage attachments.

This paper is organized into six (6) separate sections. Sections II and III introduce the analysis method for exploring the design space with a pre-populated parametric finite element model (FEM) in a DOE-based dynamics analysis using a provided outer mold line (OML). Section IV, aeroelasticity review, presents the results of the DOE FEM to demonstrate global structural stability or positive aeroelastic margin from the fluid-coupled structural response. Section V introduces critical load cases that form a part of this work and were used as the design space constraints. Conclusions are presented in Section VI.

\section{Finite Element Analysis, Structural Modeling Computer Aided Design (CAD)}

A baseline finite element model (FEM) was created consisting of a quadrilateral element dominant, high-fidelity mesh including three spars, 30 ribs, 20 stringers, rib cap stiffeners, leading edge (LE) motor pods, and wing-tip mounted engine pods. The pods contain shear webs and bulkheads for structural support. The engine pods, shown in Figure 2 and Figure 3, are attached to the main wing spars via streamline pylons to reduce stressed-skin loading, to mitigate global mode participation, and to add stiffness. The final design shown in Figure 3 resulted in a three spar configuration with pod structure reduced to a simple shear web with lateral constraint provided by an external fairing (not pictured) when installed. The semi-span model also includes a deployable flap structure containing a single spar and 24 ribs and is attached to the main wing element via implicit interpolation elements (RBE3). The model contains over 364,000 degrees of freedom with explicitly modeled primary structure (CQUAD elements) and CBEAM rib cap stiffener elements. Finite element analysis (FEA) models were rapidly developed from a generic, fully populated FEM located on commonresearchmodel.org ${ }^{6}$ and morphed to the appropriate loft and planform in HyperWorks. ${ }^{7}$ The generic FEM contains dozens of ribs and spars at each element intersection which are selectable by the user (using FEM groups) to reduce the design space as desired. This single full-featured, multi-disciplinary (stress, dynamics) FEM was morphed in span, sweep, chord thickness, and primary structure locations to explore the design space rapidly in the design of experiments environment for load-stress response, frequency content, and weight as principal design objectives. Section III details the DOE variables and their interactions.

This model suite served as the baseline for structural and aero-elastic analysis prior to design freeze and generation of a completely new vendor supplied CAD/FEM model set based on manufacturing constraints and corporate design practice. 


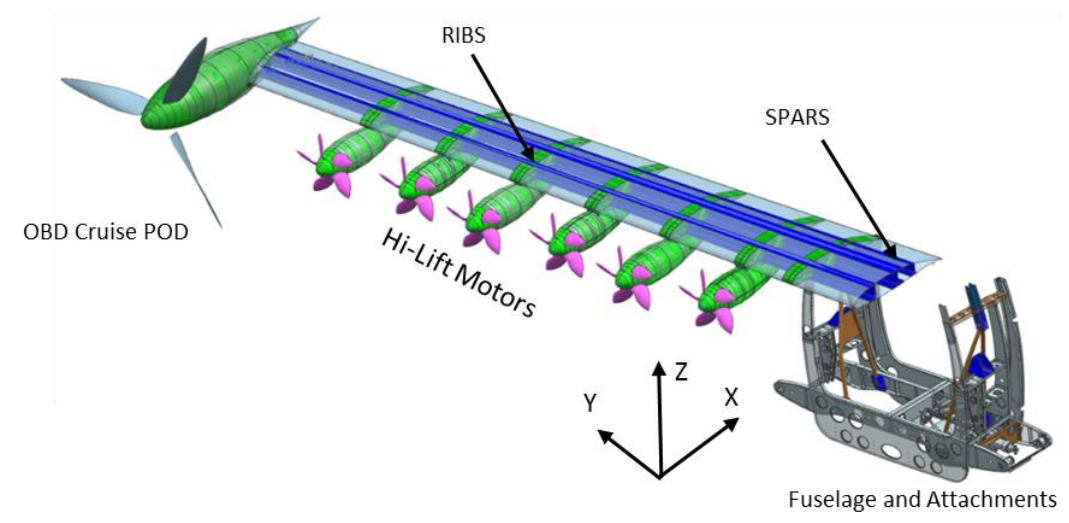

Figure 2: SCEPTOR DOE baseline wing primary structural CAD layout and attachment

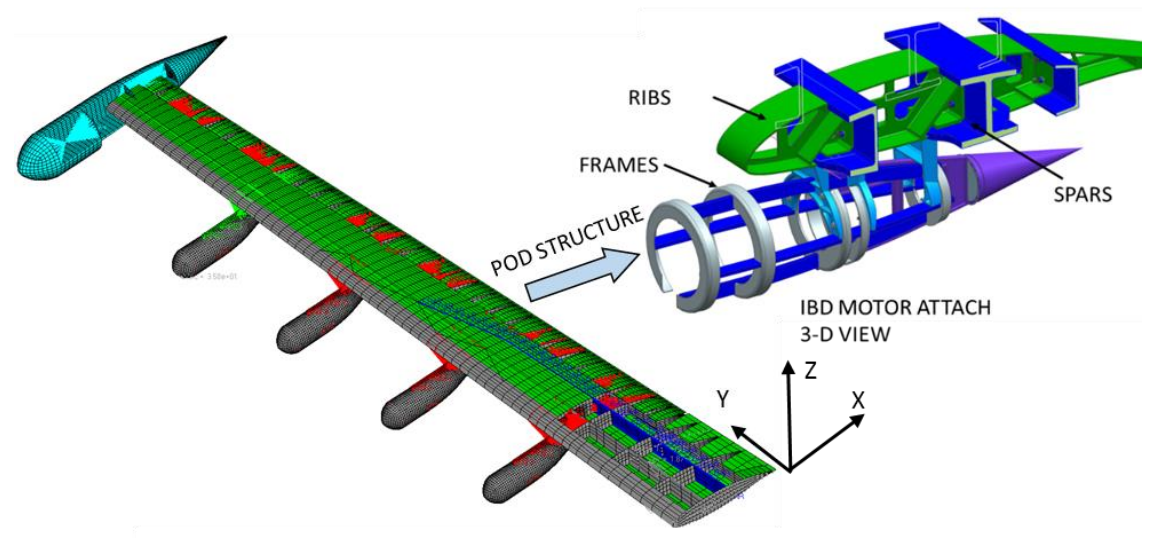

Figure 3: SCEPTOR baseline design exploration FEM and engine pods (upper skins removed for clarity)

The design process implemented evolved from a quasi-inverse design, using parametric FEM-morphing and DOE analysis to compliment a traditional manufacturing implementation and validate design space subsequent to freezing the CAD design. That is, the initial design was not initially constructed in CAD, but rather a parametrically morphed FEM which was modified for geometry and material properties based on static strength and aeroelastic stability. After design convergence with a stress and dynamics-based DOE FEM sweep, CAD models were constructed for the "production-design" of the SCEPTOR flight vehicle, and a new FEM (denoted as "CAD_X" and "FEM_X", respectively) were generated and analyzed for strength and aeroelastic behavior iteratively in a traditional manner.

The quasi-inverse approach to the process, pictured in Figure 4a, permitted the exploration of thousands of wing design candidates prior to generating costly CAD models and initiating a recursive design process. Figure $4 \mathrm{~b}$ shows the CAD-based realization from the parametric studies (e.g., the point-design freeze of the "production-design") which incorporates manufacturer-specific practices for a future production article. The chief modifications in the production model point-design being forward and aft spar load path (knee bend) to mate with existing fuselage connections and the incorporation of a stressed skin, shown in Figure 5, near the aft fuselage attach point to simplify installation and provide additional torsional stiffness. After preliminary production designs were configuration frozen, the DOE model was also converted to an all-composite material system, for internal structures like ribs and pod attachments, which retained principle load path features. This design philosophy was propagated throughout the wing structure to include skins, main spars, ribs and pod structures. 


\section{DOE EXPLORATION - FINAL CAD/FEM DESIGN}

a)

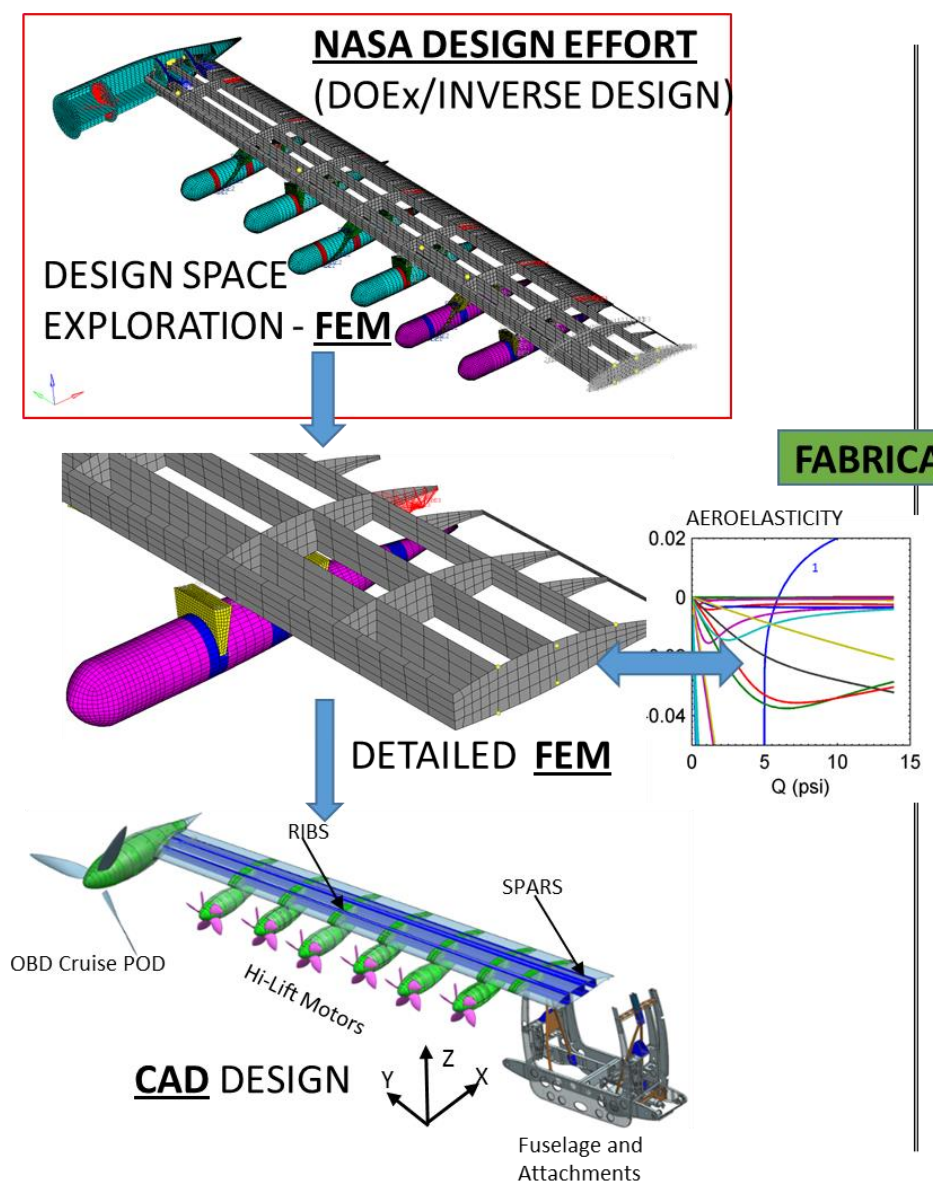

b)

\section{XPERIMENTALINC DESIGN REALIZATION}

(TRADITIONAL APPROACH)

MFGR CONSTRAINTS

- MATERIAL SYSTEMS

- MOTOR SIZING

- OML -AERO

- fuselage ATtACH- TECNAM

CAD DESIGN

C

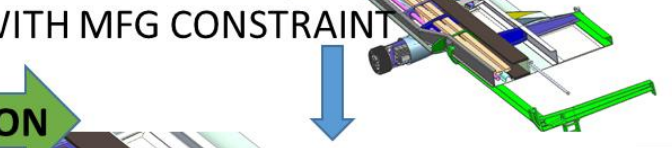

- AEROELASTICITY
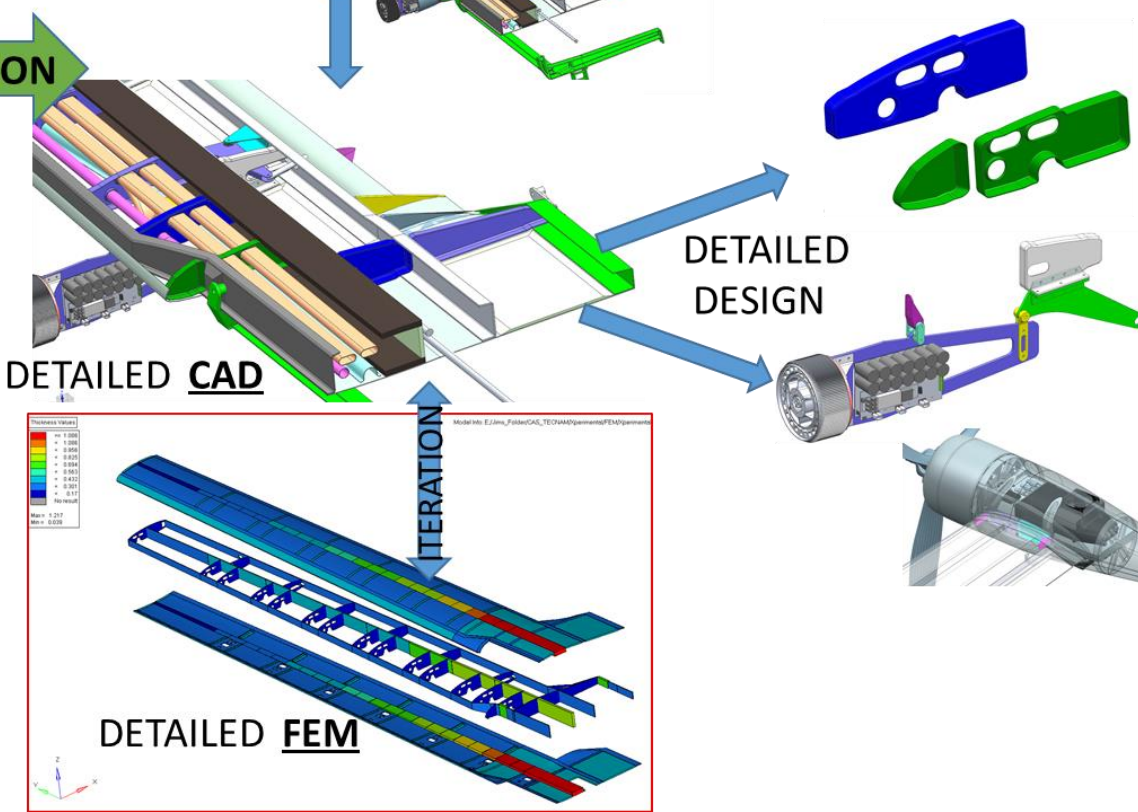

Figure 4: SCEPTOR wing primary structural layout and design process

American Institute of Aeronautics and Astronautics 
As an example, the FEM_X composite point-design realization strategy was also incorporated into the fuselage attach fittings and frames redirecting the vertical load path from main spars, through the wing skin, to a hard-back fitting interface. The wing to fuselage attachment design maintained the existing fuselage attachments for serviceability and minimizes interference with the existing structure while permitting the application of a stressed skin primary load path. Figure 5 shows the addition of a "hardback" frame-fitting incorporating a lateral sway bar (Figure 5, right). This connects the wing primary structure and reduces eccentric loads that would result from multiple indeterminate attachment points. The revised wing attachment locations are captured in the manufacturing FEM_X boundary conditions for structural analysis. Also incorporated was an aft wing skin fairing section to connect the load path from the aft fitting to the main spars internal to the wing. In this configuration the, aft wing skin region forms an integral primary load path on the intercostal (Figure 5, left) for the wing structure which determines the structural boundary conditions that affects the structural modes and increases torsional stiffness.

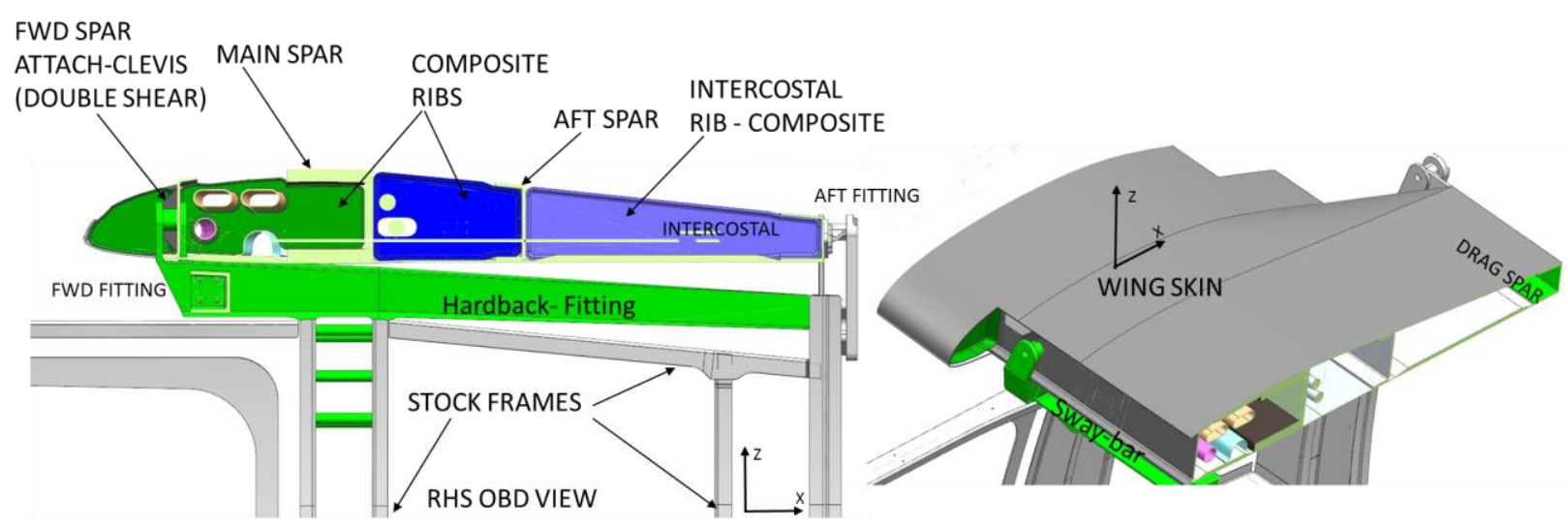

Figure 5: Wing attach manufacturing CAD_X design boundary conditions and interface, Xperimental LLC

\section{Structural Dynamics Design Space}

Aerodynamic requirements for SCEPTOR resulted in a multitude of structural solution sets when examined via a DOE method. In order to review the relative performance of each unique design, the medium-density generic FEM (Figure 3) was utilized to generate a matrix of selectable configurations. This exercise was conducted in a DOE environment to investigate the design spaces (configurations) feasible for the SCEPTOR vehicle.

Numerous configurations were generated including variable span, sweep, aspect ratio, taper ratio, panel thickness and material properties. In order to rapidly analyze the static loading and aeroelastic stability of the model set, a DOE formulation for the FEM was constructed in HyperWorks using a full factorial approach to examine the various permutations of a morphed DOE model. A sample wing FEM was modified, as shown in Figure 6, which portrays the variation in sweep, span and material properties (Young's modulus E) constructed by morphing the wing-box model, rather than re-meshing the geometry and introducing additional uncertainty.

A Quadratic Correlation Matrix (QCM) ${ }^{7}$ analysis, based on DOE variables, was used to evaluate the wing design space. The QCM is a matrix-based regression analysis of model inputs and variable sensitivities with respect to each other for a given analysis (stress, dynamics etc.). The QCM indicates the percentage of observed coupling, or correlation, between input variables as they are changed during the analysis to populate the regression variable fields and are displayed in matrix format for simplicity. The results shown herein are displayed in easily identified colorcoded-percentage format with dark blue indicating nearly a unity (identical) correlation, red indicating high negative correlation above $-50 \%$, and light blue indicating high positive correlation with other color-percentage indications scaled between extremes. These parameters were also concurrently varied during a parameter sweep for strength and natural modes, a technique commonly used in optimization strategies. ${ }^{1}$ Thousands of DOE cases were examined with two representative QCM studies shown in Table 1 that vary wing parameters based on the constraints of frequency response $(f 1-f 6)$ in the upper left corner of the table. This Table shows wing variables that have high 
coupling between modes as indicated by values approaching unity (in blue, red and yellow). Table 1 (right) shows the QCM for varied modulus and spar location as a percent of chord. Although some coupling is evident, the spar location primarily affected modes 5 and 6 whereas the effects of global modulus $\mathrm{E}$ and span are significant much earlier in the spectrum (modes 1-4). Thus, spar location was determined to have lower importance and this low sensitivity was confirmed by subsequent aeroelastic analysis of changes in spar location. Solid blue regions with QCM values of unity along the diagonal indicate values in the DOE matrix which are unchanged from the unmodified or coincident variables. Motor thrust inputs had little correlation (less than 0.1 and shown in green), which is an expected result of the linear FEM static solution utilized. Thrust coupling was not strongly evident in the QCM as expected for the linear static solution and may be more prevalent when utilizing a higher-order pre-stressed solution set (e.g. NASTRAN Solution 106) to understand the influence to the structural natural modes for future work.

The DOE parameter sweeps from the morphed FEM models were examined for variations in main spar placement and wing span simultaneously which also confirmed insensitivity of the spar placement to the fundamental natural frequencies (in modes 1 through 3 ) and aeroelastic stability for this design space. Figure 7, which is a product of the QCM solver computations, also shows a clear confirmation of the first fundamental mode frequency $\left(f_{1}\right)$ decrease with longer span or a reduction in modulus. These parametric results provide the designer with a clearer understanding of the design space and practical solution sets for each design scenario with regards to other variables like sweep, modulus and modal coupling. As a result, the leading edge spar location was retained at the $25 \%$ chord location as originally proposed. The high sensitivity of the global wing frequency response with span and modulus, however, led to the use of high modulus, unidirectional fibers in order to maintain aeroelastic margins and minimize spanwise deflections. Additionally, the design space examination shown in the surface plot illustrates the relative high sensitivity of span and much lower coupling of sweep when examining fundamental structural modes. This permitted the selection of an un-swept wing of aspect ratio 15 to allow for the reduced drag benefit of a longer span, high aspect ratio wing with stable flutter margins.

Early aeroelastic analyses, implemented with NASTRAN (solution 144, 145) ${ }^{8}$, indicates greater stability with conventional aspect ratios (9 to 10) and fewer engines. Computational Fluid Dynamics (CFD) results however show greater aerodynamic efficiencies with higher aspect ratios (greater than 11) with marginal aeroelastic stability as aspect ratio increases beyond 18. Therefore, a wing planform with an aspect ratio of 15 was selected as the baseline configuration to investigate static strength and aeroelastic performance within the DOE framework.
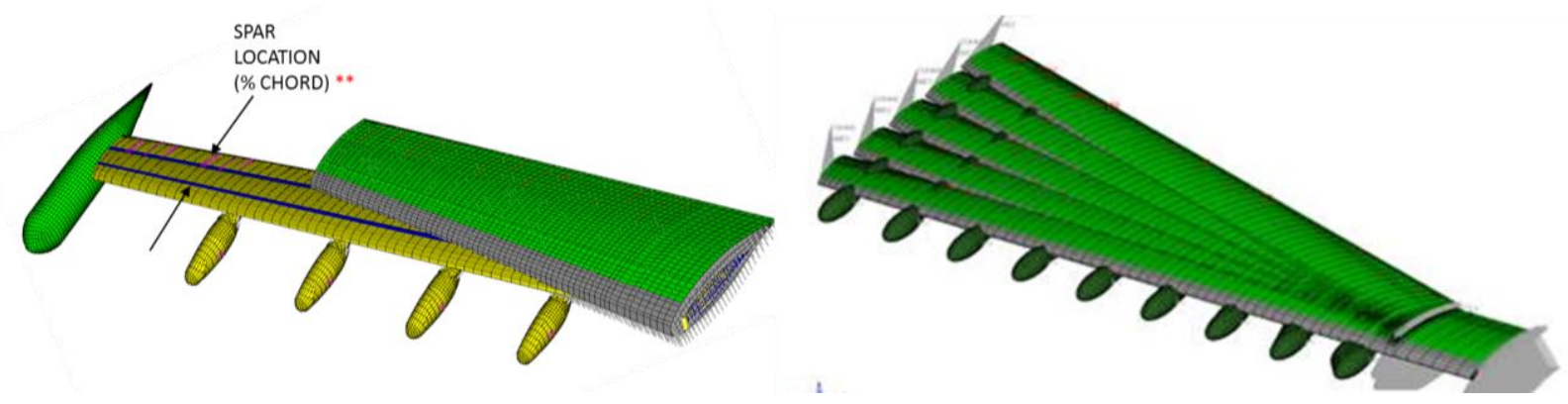

Figure 6: DOE design space examination (aspect ratio-AR, span, sweep, dihedral, spar morphed wing FEMs). Span, Spar percent chord, materials changes (left) and sweep changes (right) 
Table 1: QCM studies with DOE variables of mass, modulus (left), spar location, modulus and chord thickness (right). (Circled Regions indicate high coupling or sensitivity used for design actions.)

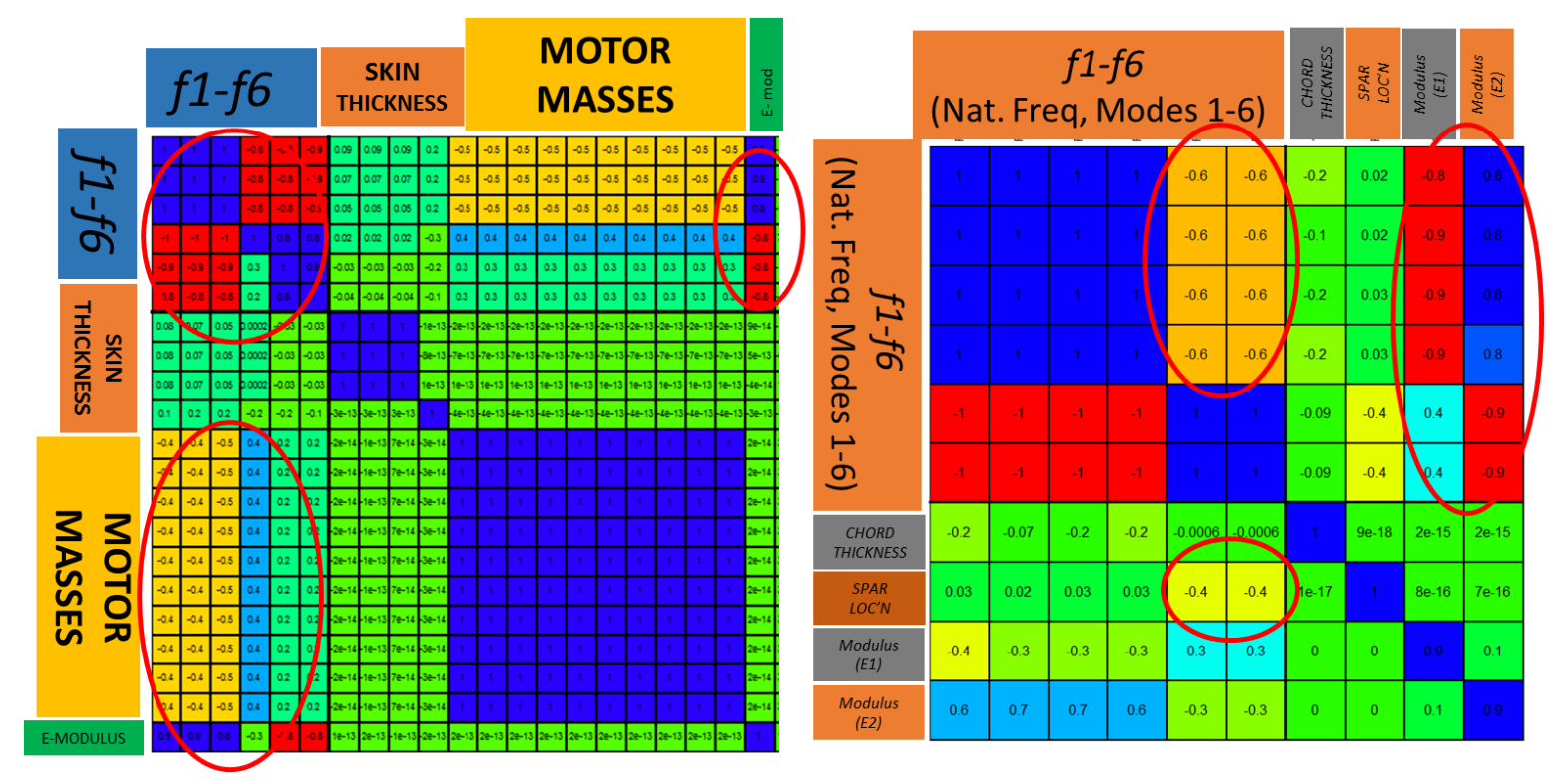

Several thousand DOE studies were conducted with two representative QCM examples of the output shown in Table 1 for selected variables shown in columns. Designers modeled the wing with conventional structural elements and varied thickness, spar locations, component quantities and modulus to form the foundation of a FEM-based DOE model to examine the structural performance. For example, as shown above in Table 1, both QCM matrices indicate strong sensitivities (circled in red) for natural modes $f_{1}$ through $f_{6}$ with motor masses, chord thickness and global material modulus. Spar location (as a percent of chord) was also strongly correlated to natural modes 5 and 6 , although not considered critical to global aeroelastic stability. Skin thickness, however did not show strong coupling for any of the first six natural modes in this case. Span and sweep QCM's were also generated (not shown) which demonstrated strong correlation to the first six natural modes as anticipated from first principles dynamics in agreement with Figure 7, right.

Eleven successive DOE FEM's were constructed to narrow down the design space with the later versions $(9,10$ and 11) being essentially equivalent. These later DOE FEM versions only had minor geometric and material parameter updates to facilitate aeroelastic studies. Permutations of each model were generated using an automated FEM morphing tool in Hyperworks. ${ }^{7}$ The study generated variations in airfoil thickness, chord, and taper ratio automatically using full factorial, Box-Behnken, and Hamersley methods to downselect wing structure design candidates for frequency response and weight minimization. Thousands of designs were produced with representative candidates that are compared in the QCM format. Studies conducted on the FEM models demonstrated the modal coupling present for these configurations and their dependencies are succinctly shown in QCM format ${ }^{7}$, which compactly depicts coupling and sensitivity of design variables. Higher aspect ratio designs are especially sensitive to modal coupling in the first bending and twist natural mode shapes (1BT). ${ }^{1}$ Strong modal coupling for this high aspect ratio design are noted for natural modes 2 and 3, shown in Figure 8, with mixed bending and torsional modes both occurring simultaneously throughout the spectrum. Higher aspect ratio designs frequently have multiple torsional mode spectra present ${ }^{5}$, indicating greater susceptibility to flutter when compared with lower aspect ratio designs that contain higher natural mode frequency ratios and larger modal separation between primary mode shapes (bend, twist). 


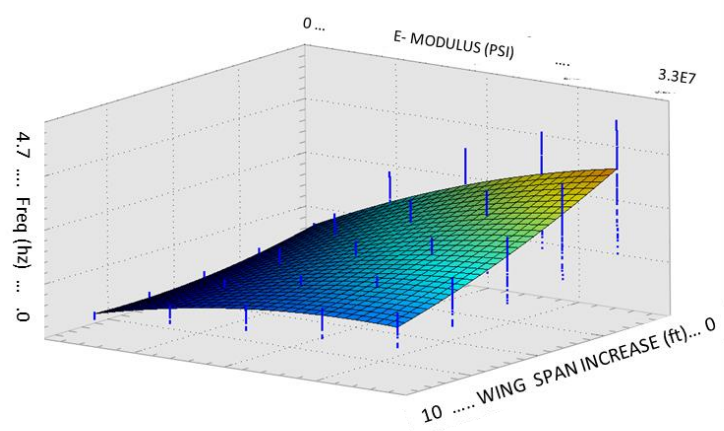

DOE PARAMETER SWEEP MODULUS AND SPAN VS FREQUENCY $\left(f_{1}\right)$

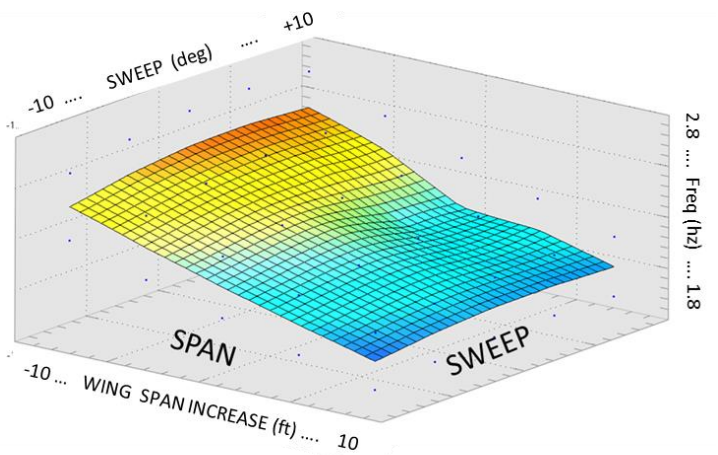

DOE PARAMETER SWEEP SWEEP AND SPAN VS FREQUENCY $\left(f_{1}\right)$

Figure 7: DOE parameter sweep of modulus versus span and frequency (left), sweep versus span and frequency (right)
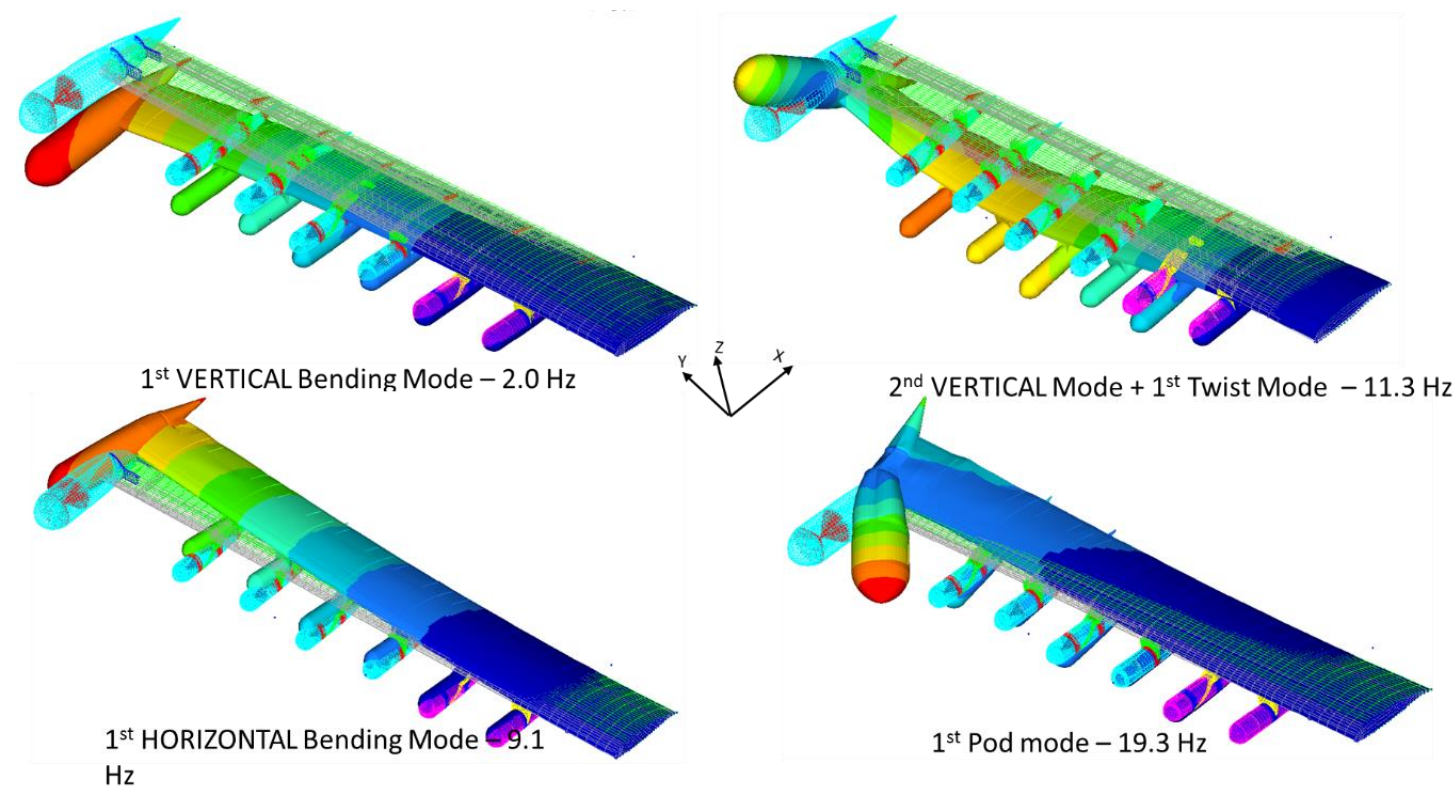

Figure 8: SCEPTOR Global Wing Bending/Twist Mode shapes 1-4 - LaRC DOE baseline FEM

Subsequent to design configuration freeze the manufacturing model set, CAD_X and FEM_X shown in Figure 9, was used to conduct final sizing exercises. The FEM_X is a model of the CAD_X design with composite spars, ribs, and sandwich core skin similar to the DOE model sets, and which contains 100,731 elements and 596,103 degrees of freedom. The notable changes from the DOE primary structure design incorporated in the FEM_X configuration included the wing attach boundary constraints, spar lamina schedules and forward/aft spar geometry which contains a knee-bend to mate to existing fuselage structure. Additionally, an aft mounted glove section was created integral to the wing skin to accommodate the existing aft fuselage attachment. The new design contains 3 spars, 23 primary ribs at pod locations and foam-core-sandwich stressed skins. The final wing structure configuration resulted in 2 ribs per pod station to provide chord -wise stability (from panel buckling and brazier crushing loads) and to permit the installation of motor wiring. 


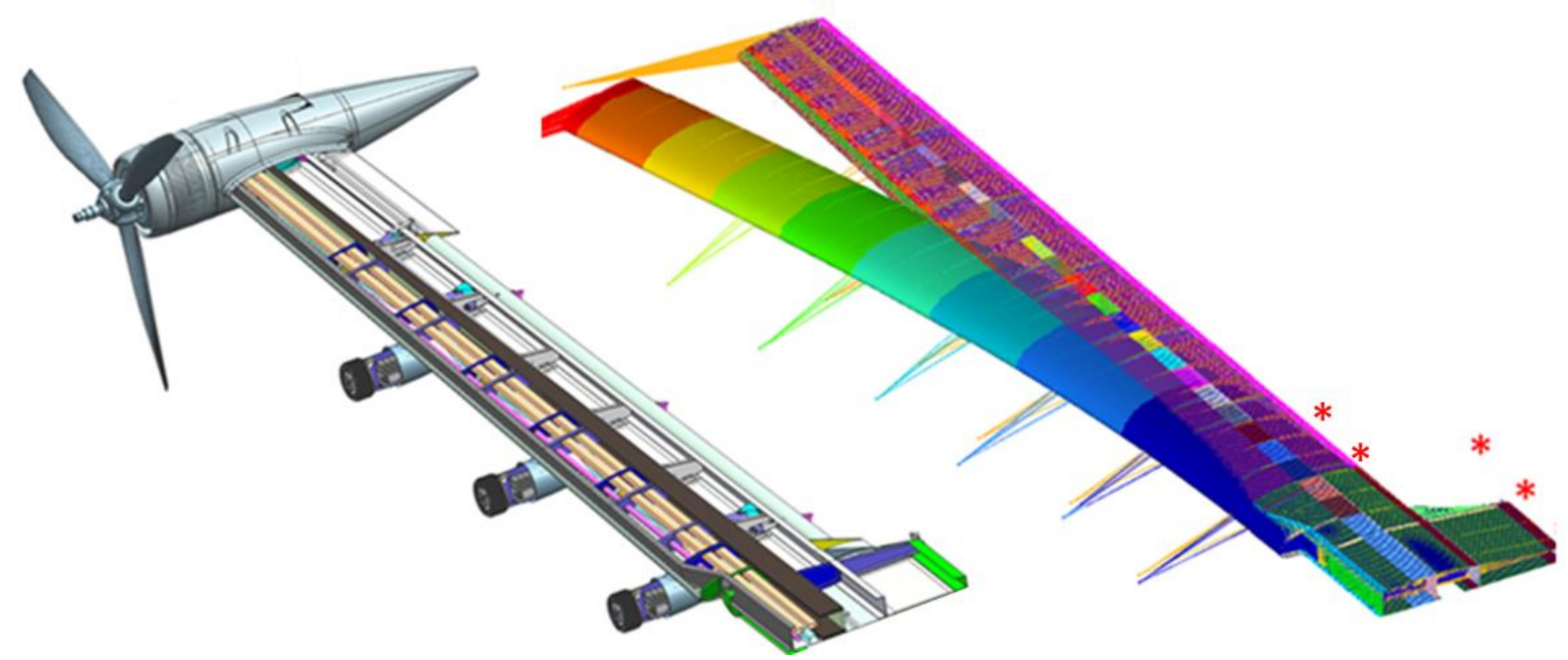

Figure 9: Xperimental LLC Manufacturing CAD_X (left) and boundary condition (*) modified FEM_X (right) natural modes (Hz) sweep with 1st horizontal bending (knife edge) mode shown.

Although the DOE FEM natural mode shapes in Figure 8 demonstrate good separation between primary mode frequency ratios it is important to note that the $3^{\text {rd }}$ global mode is a combination of vertical and twist modes which is frequently found in later modes for lower aspect ratio wing structures. More importantly, the second global natural mode ( $1^{\text {st }}$ horizontal - "knife edge"), which typically appears at higher frequency spectrum values, requires in depth analysis using CFD methods due to the inability of traditional panel codes (e.g., NASTRAN SOL144/145) to evaluate this out-of-plane aeroelastic behavior. Lower aspect ratio wings frequently exhibit bend-twist coupling between the $2^{\text {nd }}$ bending and $4^{\text {th }}$ global twist mode, later in the spectrum ${ }^{5}$, which further demonstrates the sensitive modal coupling of the SCEPTOR wing structure indicated earlier in the QCM. The effects of motor mass loading on a thin-chord, cantilevered wing with very high aspect ratio is evident in the lower principal natural frequencies. This effect implied narrower aeroelastic stability margins for this case which will be discussed in Section IV. Although aeroelastic stability can be demonstrated for the first vertical bending modes approaching $1.0 \mathrm{~Hz}$, concerns over pilot-induced oscillation (PIO) predicated that the first bending frequencies is over $2.0 \mathrm{~Hz}$. This higher frequency requirement ultimately drives the aeroelastic stability and stiffness requirement for this design, and also influences the primary structural sizing for static strength as will be discussed in further detail in Section V.

The FEM_X initially contained lower than anticipated modal response in the $1^{\text {st }}$ horizontal bending natural mode (mode 2) - annotated in Table 2, column 2, at $2.76 \mathrm{~Hz}$. This model was compared with DOE results for stiffness by varying material modulus and lamina for critical features (spars, skins) with the results shown in columns 3 through 5. A dynamics response exercise was also conducted, consisting of a mode sweep comparison for an isotropic material modulus (Aluminum, AL+) and skin-web thickness changes to FEM_X, to examine modal disparities initially encountered between DOE and FEM_X models. This material parameter sweep was conducted on both DOE11 and FEM_X model sets to investigate the disparity. 
Table 2: FEM_X natural modes sweep and DOE11 NATURAL MODES (Hz). Mode 2 Results for Parameter sweep shown in box and highlighted

\begin{tabular}{cccccc} 
Mode & FEM_X & FEM_X (AL+) & FEM_X(1/2" WEB) & FEM_X(1/2" SKINS, UNI) & DOE11 \\
1 & 1.6 & 2.35 & 1.92 & 2.79 & 2 \\
\hline 2 & 2.76 & 7.17 & 7.24 & 7.5 & 9.12 \\
\hline 3 & 8.66 & 13.17 & 9.98 & 14.45 & 11.35 \\
4 & 12.15 & 24.59 & 18.13 & 18.89 & 19.36 \\
5 & 19.25 & 31.14 & 28.55 & 33.45 & 21.03 \\
6 & 26.82 & 36.76 & 33 & 35.79 & 25.93
\end{tabular}

It was quickly determined that lower frequency for mode 2 resulted from a lack of spanwise stiffness. This result was consistent for several boundary condition modifications that reduced span to increase mode frequency (e.g., for locations shown with a “*” in Figure 9 (right)). When these additional fixed constraints were imposed, it also did not result in the higher fundamental frequency responses desired. Lamina in these regions were subsequently modified based on original DOE lamina properties to include additional unidirectional fibers in spar caps to arrest low frequency global behavior of the wing structure.

Table 2, columns 2 through 5, shows the modification of the X_FEM's material system for several options shown in columns. The X_FEM is modified to a fully homogenous material AL + (in column 2), increased spar shear web thickness (column 3), unidirectional fiber applied to skins (column 4) and unidirectional fiber applied to spars (column 5). Column 6 (DOE11) shows the modal response for a structure that contains all these modifications except for the option of aluminum. These combinations demonstrated the need for increased directional stiffness and the incorporation of spanwise unidirectional fibers in the forward and aft spars along with certain regions of the skin. Addition of unidirectional fibers spanwise in forward and aft spars was implemented to increase stiffness to ensure similarity between the FEM_X and DOE11 dynamics response. This resulted in an increase in modal frequencies of the FEM_X, more closely matching the DOE11, to provide additional confidence that the structure would be free of global aerodynamic instability. After incorporation of thicker skins and unidirectional material in spars and skin regions, the second fundamental (knife edge) mode was increased to over 7+ Hz shown in Table 2, column 5 which implies roughly a $6.5 \mathrm{X}$ increase in directional stiffness and increased aeroelastic stability margins to retain "in family" aeroelastic behavior the design space. In family behavior is characterized as having similar aeroelastic divergence and basic mode shape responses that are roughly scalable. These characteristics will be discussed in Section IV.

The resulting wing acreage lamina thicknesses are shown below in Figure 10. This design was based on the static strength requirement and modal response contribution. Spar cap thicknesses range from 0.67 inches at the root to 0.125 inches thickness at the tip. Sandwich skin panel total thickness, including core, is relatively uniform at 0.250.30 inches. Local panel buckling modes and skin compression loading have a strong correlation with rib spacing and panel thickness. Therefore, ribs were initially placed with 12-inch to 24-inch conventional spacing at each pod station to support vertical compression loads and direct aero-loads to the main spars. The SCEPTOR wing has one rib set at each pod location to reduce intra-bay buckling issues for gust loading at design limit load (DLL) condition of approximately $+3.42 /-1.37 \mathrm{~g}$ 's and to meet aerodynamic load requirements.

Manufacturing-driven design constraints also resulted in a wing structure with three spars to accommodate high torsion/bending loads and create provisions for fuselage attachments and whirl flutter abatement at motor pod locations. A continuous main spar with forward and aft spars that contain sharp knee bends permitted integration with existing fuselage interfaces. Wing skin sandwich core thicknesses were chosen based on results of the aeroelastic surveys and static loading (gust, taxi bump etc.) leveraged in part from the baseline DOE FEM results. 


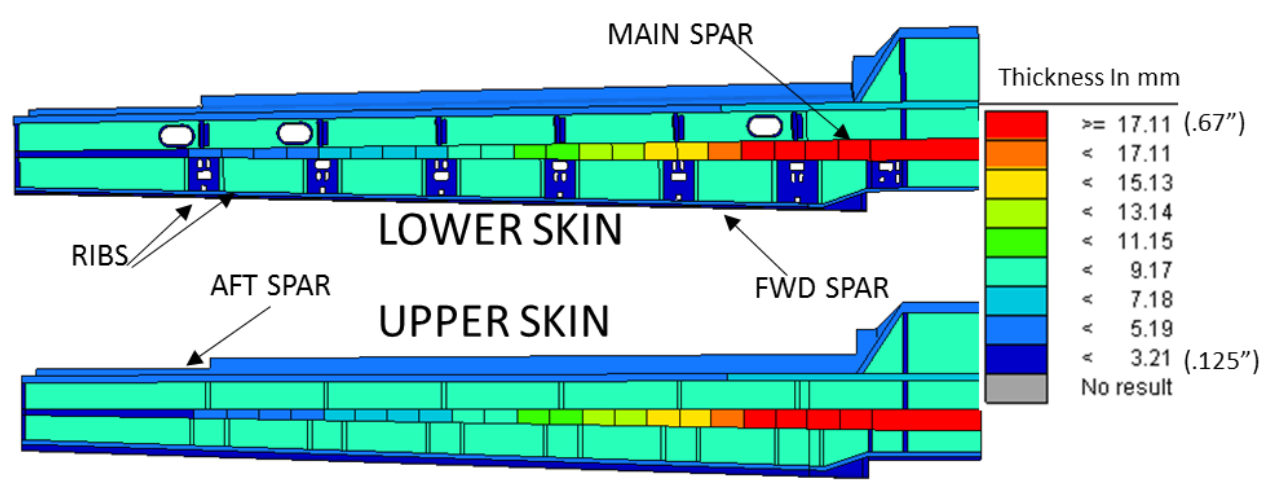

Figure 10: Final wing layout - laminate thickness (FEM_X)

\section{Aeroelastic Response, baseline DOE FEM}

The DOE SCEPTOR wing design utilizes spanwise tapered skins, spar caps, shear webs and constant rib thickness. The primary structural weight is approximately $98 \mathrm{lbs}$., not including detailed interface components and motor masses. A NASTRAN analysis was conducted using the FEM to review the global stresses and aeroelastic stability. The DOE wing and FEM_X models were found to have sufficient margin at the $1^{\text {st }}$ bending divergence mode, with a bi-furcation response at locations (1), (2) and (3) in Figure 11 for divergence modes, at dynamic pressures over $14.0 \mathrm{psi}$ as depicted in Figure 11. Instability theoretically occurs at the frequency coefficient cross over point equal to $f=0$ and is not evident here. Blue curves (1), (2) and (3) for the model sets demonstrate stability well beyond the usable flight regime. Other wing modes were stable (eigenvalue frequencies less than 0 ) for the entire flight envelope regardless of speed. For the DOE model, for example, this response represents a factor of safety for divergence of $8.0(14.0 \mathrm{psi} / 1.75)$ based on a $2 X 0.875$ psid minimum dynamic pressure $(\mathrm{Q})$ dive speed requirement ${ }^{5}$ from the FEM derived natural modes shown in Figure 8. FEM_X models (v1, v2) also demonstrate positive margins with Q greater than 14 psi although version 1 (v1) of the FEM_X had a bifurcation point (2) approximately 2 psi lower than the other models with accompanying lower knife edge mode frequency.

It was also found that aeroelastic stability was greatly reduced for AR's between 15 and 18. Initially, the design implemented an aspect ratio greater than 18 , but divergence stability margins were dramatically reduced from Q of 8 psid to roughly 2 psid dynamic pressure which are inadequate margins per AC 25.629.
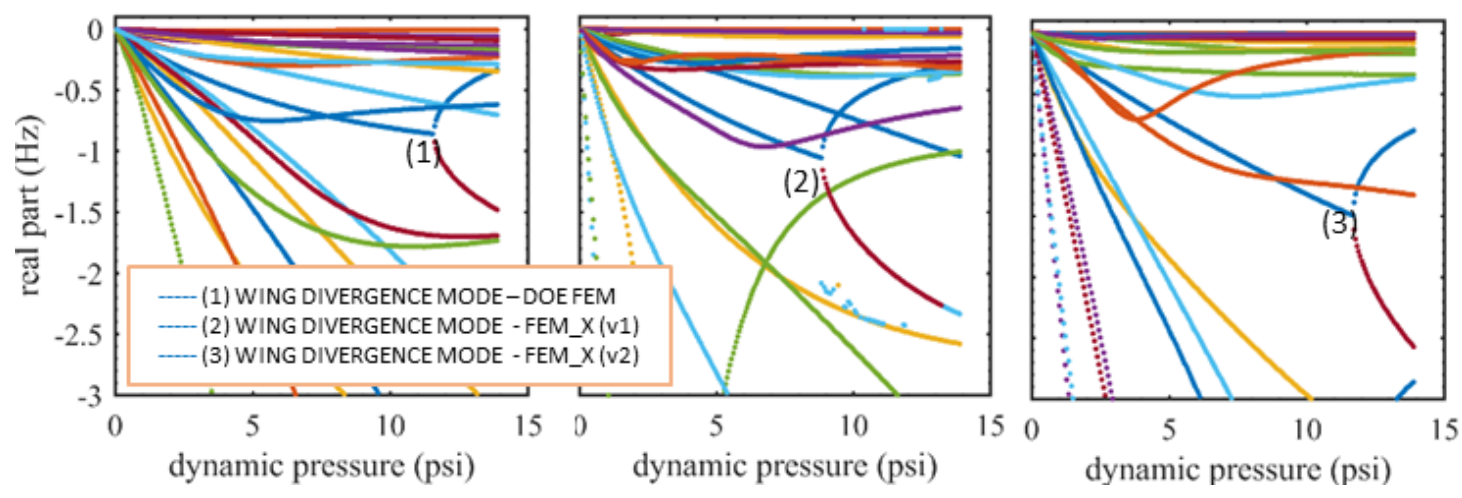

Figure 11: Dynamic pressure $Q$ vs frequency plots for DOE (left) and FEM_X wing FEM's (version 1 middle, version 2 right) design of aspect ratio 15 (LaRC-AB) showing similar divergence mode trends 
Frequency ratios of the first 6 natural modes of a structure often provide a measure of global aero-structural stability. ${ }^{5}$ Due to the outboard mounted motor pod and thin wing chord design, however the in-plane bending (knife edge mode), shown in Figure 9, became a principal design objective for mitigating structural coupling. The knife edge mode typically resides in higher modes for conventional aircraft and was also encountered in the DOE FEM's second natural mode response. Aeroelastic panel codes, such as NASTRAN solution 144, are time independent, and are not designed to evaluate in-plane aeroelastic response. This necessitated CFD-based approaches utilizing a more computationally expensive time-marching, Navier-Stokes formulation, however, these analysis were not completed in time to be included in this paper.

\section{Loads Review - Critical Cases}

Numerous load cases are documented in FAR 23 for the evaluation of a new wing structure which serves to validate the inverse approach taken. For initial exploration, a three dimensional SLD was utilized. In the interest of program efficiency, the following conditions are first considered to meet the spirit of regulations AFRC G-7123.1-001 ${ }^{9}$ flight test research requirements. These criteria are taken together as a performance based criteria similar to guidance in ASTM F $2245^{10}$ to minimize burdensome prescriptive analysis when appropriate due to the nature of a "public-use" vehicle which is not required to meet the full certification of FAR 23 regulations. FAR 23 critical load cases and related advisory circulars that were evaluated are listed here:

- Up/Down Gust (FAR 23.341) $)^{11}$

- $\quad$ Taxi Bump (FAR 23.235, 473, AC25.491)

- $\quad$ Maneuver Loads (FAR 23.337, NLT 3.8g)

- $\quad$ Ground Loads and Landing (FAR 23.473,477-9,481-5,493,497)

- Aeroelastic Response and Mode Shapes (AC 25.629-1B)

The unique $\mathrm{C}_{1}$ from the CFD results in Figure 12 demonstrate non-zero tip $\mathrm{C}_{1}$ in the powered condition (upper left) and the applied cruise loading in a more conventional elliptical $\mathrm{C}_{1}$, unpowered condition (lower figures). The basic envelope load (V-n) diagram is also represented (upper right).

Due to the unique SLD of the SCEPTOR wing configuration, the power-on condition of the distributed propulsion leading edge concept experiences a much higher than normal lift coefficient, approaching $C_{1}=5$, shown in Figure 12, upper left, than the unpowered state. This is similar to the Custer Channel Wing configuration ${ }^{12}$ and demonstrates concept validity for the load sizing. In the unpowered state, $\mathrm{C}_{\operatorname{lmax}}$ is approximately 1.0 with the flap stowed, as shown in the lower left of Figure 12, with nacelle locations demonstrating some discontinuity. These loads were used as the basis for the gust and dive envelope sizing. 


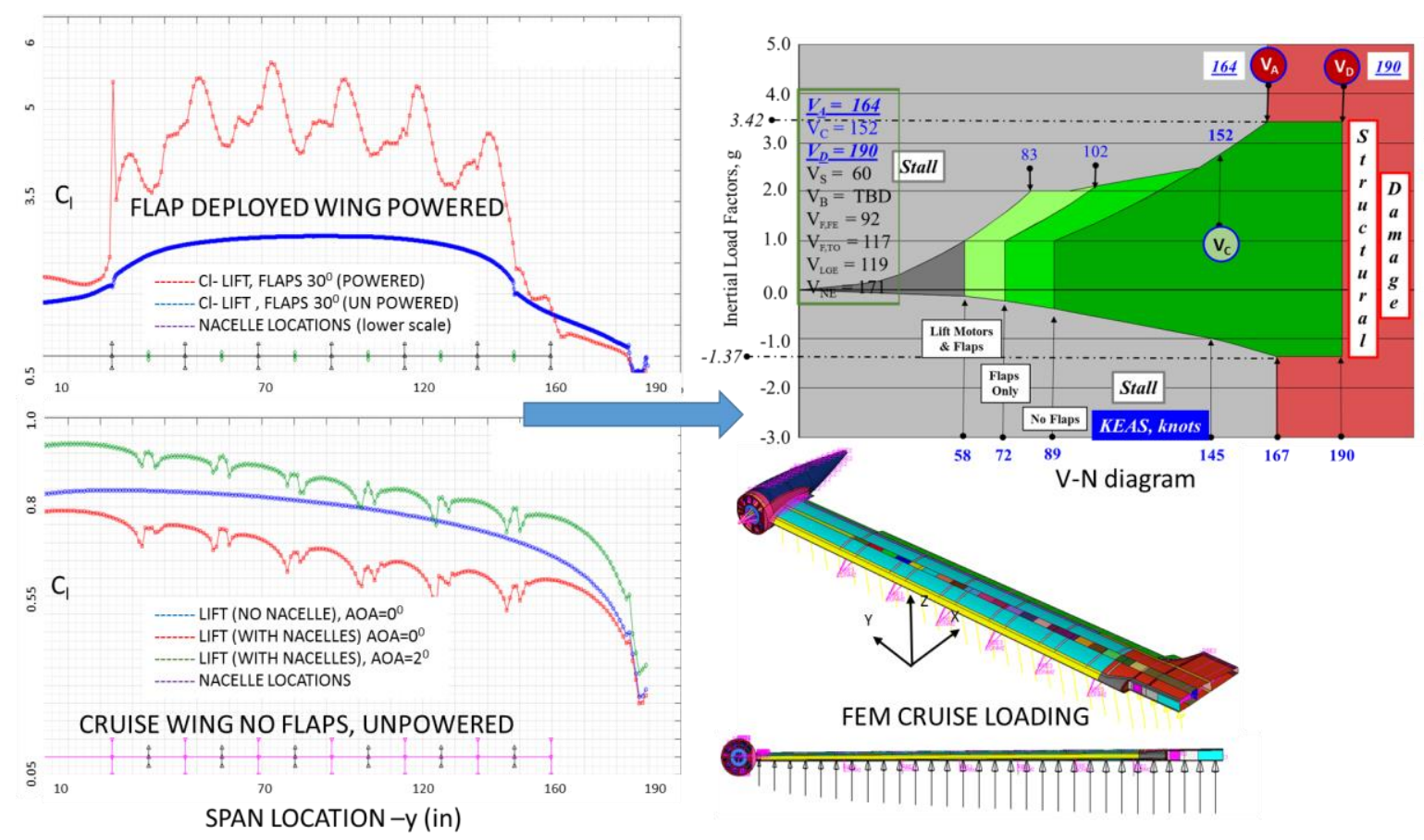

Figure 12: SCEPTOR predicted $C_{l}$ (left), FEM_X with cruise loading (lower right), V-n diagram

Additionally considered in this unique configuration are loads resulting from the inertia contribution from motor placement. The requirement for a stiff outboard pod connection, to reduce inertia and whirl flutter contribution, is in sharp contrast with conventional construction with isotropic materials ${ }^{13,14}$ for this region. The design goal is to avoid a global flutter response and high vertical inertial loading during taxi or hard landing conditions. Additionally, the outboard position of the propeller also creates concern for approach slip or crosswind angles which may cause contact with the ground during a hard landing condition that flexes the wing downward and these design conditions were simultaneously considered. Interaction of the propeller with the wing structure (AC25.905) and with ground surfaces are considered critical cases and were examined separately for compliance to operational requirements referenced above for ground handling, blade-out scenarios and preliminary investigation for whirl flutter behavior.

\section{Concluding Remarks}

SCEPTOR is a novel aircraft design which utilizes a multi-propulsor configuration consisting of 14 leading edge mounted electric motor pods. This configuration enables the aircraft to operate more efficiently using a high aspect ratio wing, which reduces wing area and lift induced drag at the expense of aeroelastic stability. The initial design space examinations with a generic DOE FEM included exploration of span, sweep, spar placement, internal topology (number of ribs, spars, stringers etc.) and material properties. Inboard motor mass loading and dynamics contributions were considered in the wing design and were not primary drivers for global aeroelastic margins. This study found that modal coupling was increased and resulting aeroelastic stability greatly reduced for AR's between 15 and 18. Therefore, a more conservative selection was made to use an aspect ratio of approximately 15 for the SCEPTOR wing structure to benefit global aeroelastic stability and localized whirl flutter behavior.

Early SCEPTOR wing designs targeted higher aspect ratios to generate maximum lift with minimum drag. This new design includes composite skin panels to reduce manufacturing cost and to minimize drag due to the inherent ability of composites to readily produce compound curvatures required for SCEPTOR aerodynamics. The quasi-inverse analysis approach used for this composite wing-box structure, which uses a parametric FEM for DOE studies rather than CAD models, demonstrates positive static and aeroelastic load margins. The preliminary wing design has been completed and is presently in detailed design for first article production. 
The SCEPTOR primary wing structure was developed using a quasi-inverse design approach to establish a performance-based loads compliance which will be examined on test article components in the 2017 timeframe. Rather than the traditional approach of designing CAD-based wing structure solid models from a loft, followed by a rigorous and time consuming analysis feedback loop, a single detailed multi-discipline FEM was leveraged to explore dynamics and aeroelastic response in an automated fashion. The result of this DOE study contributed to generation of the detailed manufacturing CAD/FEM. This parallel design/analysis approach provided additional confidence in design performance and a basis for dynamics and strength correlation for future model sets. Aeroelastic and dynamics stability were principal design objectives, due to the high aspect ratio and flexible nature of this wing, and were evaluated "up-front" as a quasi-inverse methodology. This inverse approach parallels the traditional re-design and analyze cycle with a full-featured morphable FEM tool, which greatly reduced development uncertainty while complying with selected performance based load cases prior to generating CAD data.

During development, it was discovered that the original DOE FEM modal performance demonstrated significantly higher frequency $1^{\text {st }}$ horizontal bending (knife edge) mode than the FEM_X. This discovery led to investigation of stiffness differences between the DOE and FEM_X model sets. Modifications to the forward and aft spar of FEM_X, in the form of spanwise unidirectional fibers, improved directional stiffness to ensure agreement between model sets which ultimately determined laminate sequencing for the first article design. These modifications also preserved original DOE stability margin targets to retain in-family aeroelastic stability characteristics. During the design process, it became increasingly clear that the high wing-loading of a thin airfoil section and leading-edgemounted propulsor masses presents a significant design challenge to demonstrate the strength and dynamic stability of the design. Strength margins for basic load cases included gust, taxi bump and hard landings with resulting operational limitations that may be imposed on crosswind or ground operations (taxi bump, ground handling etc.) to satisfy safety flight testing requirements.

The global aeroelastic response of the wing, and related global stiffness of the wing and motor pod structures, became the principal design metric for this configuration which also prompted high fidelity examination of the fluid structure interaction using CFD-based structural dynamics tools. The requirement for increased aeroelastic stiffness resulted in the addition of another main wing spar, thicker wing skin acreage and addition of unidirectional fiber in forward and aft spar caps to enhance local stiffness. Correlation of the DOE FEM and FEM_X models used in the model validation process proved invaluable for evaluating modal performance and directly impacted the final manufacturing design. This collaborative, quasi-inverse methodology led to a more robust, dynamically stable configuration with predictable aeroelastic margins.

\section{Acknowledgements}

This work was funded by the Convergent Aeronautical Solution (CAS) project under the Transformative Aeronautics Concepts (TAC) program project for Aeronautic Research Mission Directorate at NASA. The collaboration provided by Xperimental Inc, San Luis Obispo CA and the NASA-LaRC Aeroelasticity Branch (Bret Stanford) in providing final manufacturing CAD_X/FEM_X designs and developing aeroelastic analytical requirements respectively, for the above research is greatly appreciated. 


\section{REFERENCES:}

1 Jutte, C., Stanford, B., Wieseman, C., and Moore, J., "Aeroelastic Tailoring of the NASA Common Research Model via Novel Material and Structural Configurations," AIAA SciTech Conference, National Harbor, Maryland, January 13-17, 2014.

2 Bradley, M., and Droney, C., "Subsonic Ultra Green Aircraft Research: Phase I Final Report," NASA CR-216847, NASA Langley Research Center, Hampton, Virginia, 2011.

3 Stoll, A., Bevirty, J., Moore, M., and Fredericks, W., "Drag Reduction through Distributed Electric Propulsion," Aviation Technology, Integration, and Operations Conference, June 16-20, 2014, Atlanta, Georgia.

4 Anon., "Minimizing the Hazards from Propeller Blade and Hub Failures," FAA Advisory Circular AC-25.905, http://www.faa.gov/regulations_policies/advisory_circulars/index.cfm/go/document.information/documentID/22 675, Washington, DC, September 27, 2000.

5 Anon., “Aeroelastic Stability Substantiation of Transport Category Airplanes,” FAA Advisory Circular AC25.6291 A.

http://www.faa.gov/regulations_policies/advisory_circulars/index.cfm/go/document.information/documentID/22 660, Washington, DC, July 23, 1998.

6 Vassberg, J., DeHaan, M., Rivers, S., and Wahls, R., "Development of a Common Research Model for Applied CFD Studies," AIAA Applied Aerodynamics Conference, Honolulu, Hawaii, August, 2008.

7 "HyperWorks," Altair Engineering, Troy, Michigan, www.altair.com, 2015.

8 Anon., "MSC Nastran 2012.2 quick reference guide," MSC Software Corporation, Santa Ana, California, www.mscsoftware.com, 2012.

9 Anon., "Aircraft Structural Safety of Flight Guidelines," AFRC G7123.1, Armstrong Flight Research Center Edwards AFB, California, 2014.

10 Anon., "Standard Specification for Design and Performance of a Light Sport Airplane," ASTM F2245-16, http://www.astm.org/Standards/F2245.htm, West Conshohocken, PA, 2016.

11 Anon., Title 14 Code of Federal Regulation, Part 23 "Airworthiness Standards: Transport Category Airplanes," Subpart C "Structure," Electronic Code of Federal Regulations, http://www.faa.gov/regulations_policies/faa_regulations/, Washington, DC, 2016.

12 Pasamanick, J., "Langley Full-Scale-Tunnel Tests of the Custer Channel Wing Airplane," NACA RM L53A09, National Advisory Committee for Aeronautics, NASA Langley Research Center, Hampton, Virginia, April 1953.

13 Anon., "Metallic Materials Properties Development and Standardization (MMPDS)," Battelle Memorial Institute, Metallic Materials Design Data Acceptable to Government Procuring or Certification Agencies, https://global.ihs.com/, April, 2015.

14 Niu, M., “Airframe Structural Design,” Hong Kong Conmilit Press, Hong Kong, 1988. 